ESAIM: PROCEEDINGS, October 2007, Vol. 22, 169-174

Gabriel Caloz \& Monique Dauge, Editors

\title{
TOWARDS A SELF-ADAPTIVE PARAMETERIZATION FOR AERODYNAMIC SHAPE OPTIMIZATION
}

\author{
Régis Duvigneau ${ }^{1}$, Badr Abou El Majd ${ }^{1}$ and Jean-Antoine Désidéri ${ }^{1}$
}

\begin{abstract}
In parametric shape optimization, results usually depend on the choice of the parameterization. In order to reduce this critical dependency, a self-adaptive parameterization methodology is developed, that adapts an initial and perhaps naïve parameterization to the problem studied, on the basis of a first approximation of the optimum shape. The proposed approach is studied in the framework of the Bézier curve representation and the Free-Form Deformation (FFD) approach. It is first applied to a geometrical arc reconstruction problem and then to a three-dimensional shape optimization problem in aerodynamics.
\end{abstract}

Résumé. En optimisation de forme paramétrique, les résultats dépendent généralement du choix de la paramétrisation. Afin de réduire cette dépendance critique, une méthode de paramétrisation auto-adaptative est développée, qui permet d'adapter au problème étudié une paramétrisation initiale et peut-être naïve, sur la base d'une première approximation de la forme optimale. Cette approche est étudiée dans le cadre d'une représentation par courbes de Bézier et par l'approche des boîtes englobantes. Elle est appliquée tout d'abord à un problème géométrique de reconstruction d'un arc, puis à un problème d'optimisation de forme tridimensionnelle en aérodynamique.

\section{INTRODUCTION}

In parametric shape optimization, a geometrical shape representation has to be chosen a priori, such as the use of Bézier curves for instance. This task is called parameterization. This choice determines a subspace in which the search of the optimum shape is performed. This strategy has several advantages, such as:

- the reduction of the dimension of the problem, that is mandatory for stochastic optimization for instance ;

- the control of the smoothness of the shapes, that is necessary for numerical calculations and most realistic applications.

However, this strategy drastically reduces the set of shapes that can be reached by the optimization procedure. Then, the optimum shape found usually depends on the parameterization. The use of a parameterization that is not well adapted to the optimization problem can yield a low fitness optimum shape. Hence, in order to reduce this critical dependency, a self-adaptive parameterization methodology is developed, that adapts an initial and perhaps naïve parameterization to the problem studied, on the basis of a first approximation of the optimum shape.

${ }^{1}$ INRIA OpALE Project-Team, 2004 route des lucioles, BP 93, 06902 Sophia-Antipolis, FRANCE (www-sop.inria.fr/opale)

(c) EDP Sciences, SMAI 2007 


\section{Self-AdAPtive PARAMETERIZATION}

\subsection{Bézier curve}

One proposes to modify some characteristics of the parameterization to adapt it to the particular problem studied. For instance, consider an airfoil shape described by a Bézier curve:

$$
P(t)=\sum_{i=0}^{n} B_{n}^{i}(t) P_{i}
$$

where $t \in[0,1]$ and $\left\{B_{n}^{i}\right\}_{i=0, \ldots, n}$ are the Bernstein polynomials of degree $n$. The coordinates of the control points $P_{i}=\left(\begin{array}{l}x_{i} \\ y_{i}\end{array}\right)$ can be considered as design variables during the optimization procedure. Since an airfoil is a rather thin body, only ordinates $\left\{y_{i}\right\}_{i=0, \ldots, n}$ are usually taken into account during the optimization, whereas abscissae $\left\{x_{i}\right\}_{i=0, \ldots, n}$ are frozen. Then, one proposes to consider the abscissae $\left\{x_{i}\right\}_{i=0, \ldots, n}$ as adaption variables that can be used to control the characteristics of the parameterization [3].

Initially, the abscissae $\left\{x_{i}\right\}_{i=0, \ldots, n}$ can be uniformly distributed. Once a first approximation of the optimum shape has been found by modifying the ordinates $\left\{y_{i}\right\}_{i=0, \ldots, n}$, new abscissae $\left\{x_{i}\right\}_{i=0, \ldots, n}$ are defined, that are better adapted to the problem, before a second optimization step is carried out. Actually, the abscissae are defined in such a way that an adaption cost functional is minimized, that measures the uneffectiveness of the current parameterization, with the constraint that the current shape remains unaltered. This constraint is introduced to benefit from the optimization path already performed.

For a given set of abscissae $\left\{x_{i}\right\}_{i=0, \ldots, n}$, consider the ordinates $\left\{y_{i}\right\}_{i=0, \ldots, n}$ for which the shape is the leastsquares approximation of the current shape. Then, the new abscissae $\left\{x_{i}\right\}_{i=0, \ldots, n}$ are chosen in order to minimize the total variation of the corresponding ordinates $\left\{y_{i}\right\}_{i=0, \ldots, n}$ :

$$
T V\left(\left\{y_{i}\right\}\right)=\sum_{i=1}^{n}\left|y_{i}-y_{i-1}\right| \approx \int_{0}^{1}\left|y^{\prime}(t)\right| d t
$$

where $y(t)$ is interpolating the control points. This criterion is introduced to regularize the control points polygon. This choice is justified by the fact that the optimization process yields a highly irregular control points polygon [2].

\subsection{Free-Form Deformation (FFD)}

For complex three-dimensional problems, such as those encountered in aerodynamics, the Free-Form Deformation (FFD) approach [6] is adopted. It consists in defining a lattice embedding the shape and a local coordinate system $(\xi, \eta, \zeta) \in[0,1] \times[0,1] \times[0,1]$ attached to this lattice. Then, the displacement of a point $q$ inside the lattice is described by a third-order Bézier tensor product:

$$
\Delta q=\sum_{i=0}^{n_{i}} \sum_{j=0}^{n_{j}} \sum_{k=0}^{n_{k}} B_{i}^{n_{i}}\left(s_{q}\right) B_{j}^{n_{j}}\left(t_{q}\right) B_{k}^{n_{k}}\left(u_{q}\right) \Delta P_{i j k},
$$

where $\left(s_{q}, t_{q}, u_{q}\right)$ results in a mapping of the coordinates $\left(\xi_{q}, \eta_{q}, \zeta_{q}\right)$ of $q$ in the lattice. Usually, this mapping is defined as the identity. The weighting coefficients $\Delta P_{i j k}$ are considered as design variables. Finally, the FFD approach allows an easy deformation of an object, regardless of the representation of this object. Figure (1) depicts the FFD lattice built around a wing and figure (2) shows the deformation resulting from a control points displacement (plain markers correspond to frozen control points whereas empty markers are control points moving vertically). 


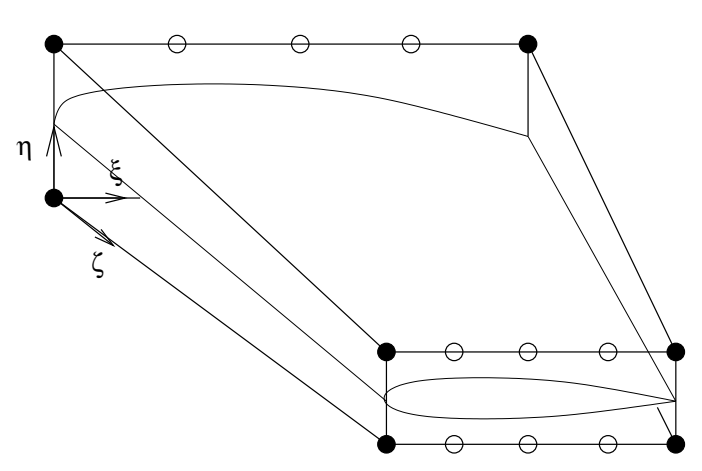

Figure 1. Initial FFD lattice.

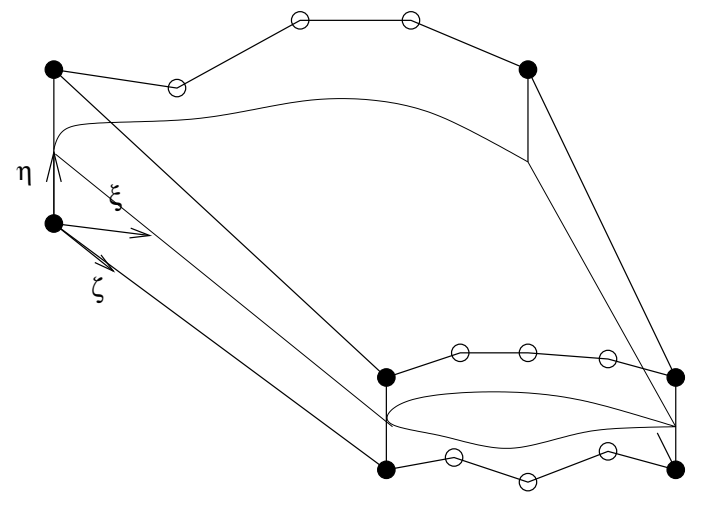

Figure 2. Deformed FFD lattice.

One proposes to consider the mapping that produces $\left(s_{q}, t_{q}, u_{q}\right)$ from the local coordinates $\left(\xi_{q}, \eta_{q}, \zeta_{q}\right)$ as adaption variables [5]. The mapping is expressed for each direction using the Bernstein polynomials basis :

$$
\begin{gathered}
s=\phi(\xi) \quad t=\psi(\eta) \quad u=\theta(\zeta) . \\
\phi(\xi)=\sum_{i=0}^{n_{i}^{\prime}} B_{i}^{n_{i}^{\prime}}(\xi) \phi_{i} \quad \psi(\eta)=\sum_{j=0}^{n_{j}^{\prime}} B_{j}^{n_{j}^{\prime}}(\eta) \psi_{j} \quad \theta(\zeta)=\sum_{k=0}^{n_{k}^{\prime}} B_{k}^{n_{k}^{\prime}}(\zeta) \theta_{k} .
\end{gathered}
$$

Finally, weighting coefficients $\left(\phi_{i}\right)_{i=0, \ldots, n_{i}^{\prime}},\left(\psi_{j}\right)_{j=0, \ldots, n_{j}^{\prime}}$ and $\left(\theta_{k}\right)_{k=0, \ldots, n_{k}^{\prime}}$ are considered as adaption variables. The adaption cost functional is inspired from the previous case (Bézier curves) and measures the irregularity of the deformation:

$$
\mathcal{J}_{A D}=\frac{1}{n_{i} n_{j} n_{k}} \sum_{i=1}^{n_{i}} \sum_{j=1}^{n_{j}} \sum_{k=1}^{n_{k}}\left\|\overline{\bar{\nabla}} \delta P_{i j k}\right\| \approx \int_{0}^{1} \int_{0}^{1} \int_{0}^{1}\|\overline{\bar{\nabla}} \delta P(\xi, \eta, \zeta)\| d \xi d \eta d \zeta
$$

where $\delta P(\xi, \eta, \zeta)$ is interpolating the weighting coefficients. $\left\|\overline{\bar{\nabla}} \delta P_{i j k}\right\|$ is the Froebenius norm of the gradient tensor estimated over the elementary volume of indices $i j k$. The minimization of the adaption cost functional is subject to the constraint that the shape remains unaltered in a discrete least-squares sense. Thus, for a given mapping, the control points displacement is obtained by minimizing:

$$
\mathcal{J}_{L S}=\sum_{n=1}^{N} \frac{1}{2}\left[\Delta q_{\text {new }}^{n}-\Delta q_{\text {old }}^{n}\right]^{2} \delta S^{n}
$$

where $\Delta q_{\text {new }}^{n}$ and $\Delta q_{\text {old }}^{n}$ represent the displacements of the mesh node $q^{n}$ for the FFD deformations that correspond respectively to the new mapping and the current mapping. $\delta S^{n}$ is a weighting coefficient expressing the discrete integration over the shape surface. Then, the adaption process consists in determining the adaption variables $\left(\phi_{i}\right)_{i=0, \ldots, n_{i}^{\prime}},\left(\psi_{j}\right)_{j=0, \ldots, n_{j}^{\prime}}$ and $\left(\theta_{k}\right)_{k=0, \ldots, n_{k}^{\prime}}$ to minimize the adaption cost function $\mathcal{J}_{A D}$, that is evaluated after the least-squares projection. 


\section{Applications}

\subsection{Geometrical model problem}

The proposed method is first applied to a geometrical problem arising from the calculus of variations [4]. It consists in reconstructing an arc by minimizing the following cost function:

$$
\mathcal{J}_{O P T}=\frac{p^{\alpha}}{\mathcal{A}}
$$

where $\alpha>1$ is a positive real number. $p$ and $\mathcal{A}$ are the pseudo-length of the arc and the pseudo-area below the arc, defined by:

$$
p=\int_{0}^{1} \sqrt{x^{\prime 2}(t)+y^{\prime 2}(t)} \omega(t) d t \quad \mathcal{A}=\int_{0}^{1} y(t) x^{\prime}(t) \omega(t) d t .
$$

$\omega(t)$ is a positive and adjustable function. It was shown in [4] that given a shape for which $y$ is a smooth function of $x$ admitting one and only one extremum, $\alpha$ and $\omega(t)$ can be set uniquely so that $\mathcal{J}_{O P T}$ is a unimodal function of $y(t)$ (for fixed $x(t)$ ) and its unique minimum is realized by the given shape.

The shape optimization procedure including the adaption method is applied to this problem [3]. Here, $\alpha$ and $\omega(t)$ are chosen in such a way that the solution corresponds to a circular $\operatorname{arc}(\alpha=2$ and $\omega(t)=1)$, for which the theorical minimum value is $2 \Pi$. The arc is successively parameterized by Bézier curves of increasing degrees. The cost function values obtained with respect to the degree are depicted in figure (3).

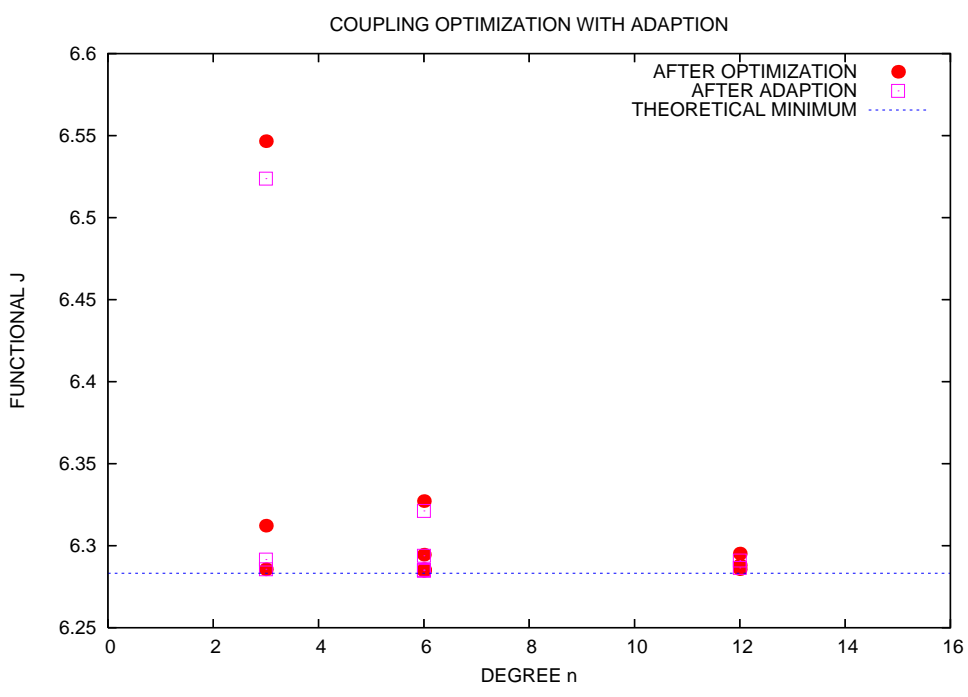

Figure 3. Results for the model problem : cost function values obtained after a single optimization and then after adaption+optimization, for different parameterization degrees.

Using a curve of degree 3 whose control points abscissae are uniformly distributed, poor results are obtained after a single optimization (about 6.55). This value is slightly decreased after adaption thanks to the leastsquares fitting. A second optimization using the adapted parameterization yields a significant improvement (about 6.31). If a third adaption + optimization step is performed, a cost function value very close to the theorical minimum is obtained. Similar results are observed using a curve of higher degree. These results show that an adapted parameterization of degree 3 allows to reach a shape of better fitness than a naïve parameterization of degree 6 or even 12 . 


\subsection{Aerodynamic shape optimization}

The proposed method is now faced with the aerodynamic optimization of the shape of a wing of a business aircraft [1] (courtesy of Piaggio Aero Ind.). The compressible flow is modeled by the Euler equations, which are solved using a finite-volume approach on unstructured meshes. The shape deformation is parameterized using the FFD approach described above. Figure (1) shows the wing shape embedded in the FFD lattice. The optimization procedure relies on the Multi-directional Search Algorithm (MSA) from Torczon [7], which is a derivative-free method similar to the well-known Nelder-Mead simplex method, but designed for parallel computations. The aim of the optimization is the reduction of the drag, including a constraint on the lift.

Optimization exercises are carried out using a coarse parameterization (8 d.o.f.), a medium one (20 d.o.f.) and a fine one (32 d.o.f.), with and without adaption. For the tests with adaption, the parameterization adaption procedure is performed every ten iterations, yielding a regular parameterization update until convergence of both optimization and adaption procedures. This strategy promotes the avoiding of local minima. Figure (4) shows the evolution of the cost function using a coarse parameterization with and without adaption and a medium parameterization without adaption. As can be observed, the use of the adaption procedure yields a faster convergence towards a shape of better fitness. Finally, the use of a coarse but adapted parameterization is more effective than the use of a medium but naïve one. The results obtained using a medium parameterization (figure (5)) exhibit the same behavior. The optimum shapes found using the initial parameterization and the adapted parameterization are depicted in figure (6). As can be observed, the fitness improvement thanks to adaption corresponds to very slight modifications of the shape.

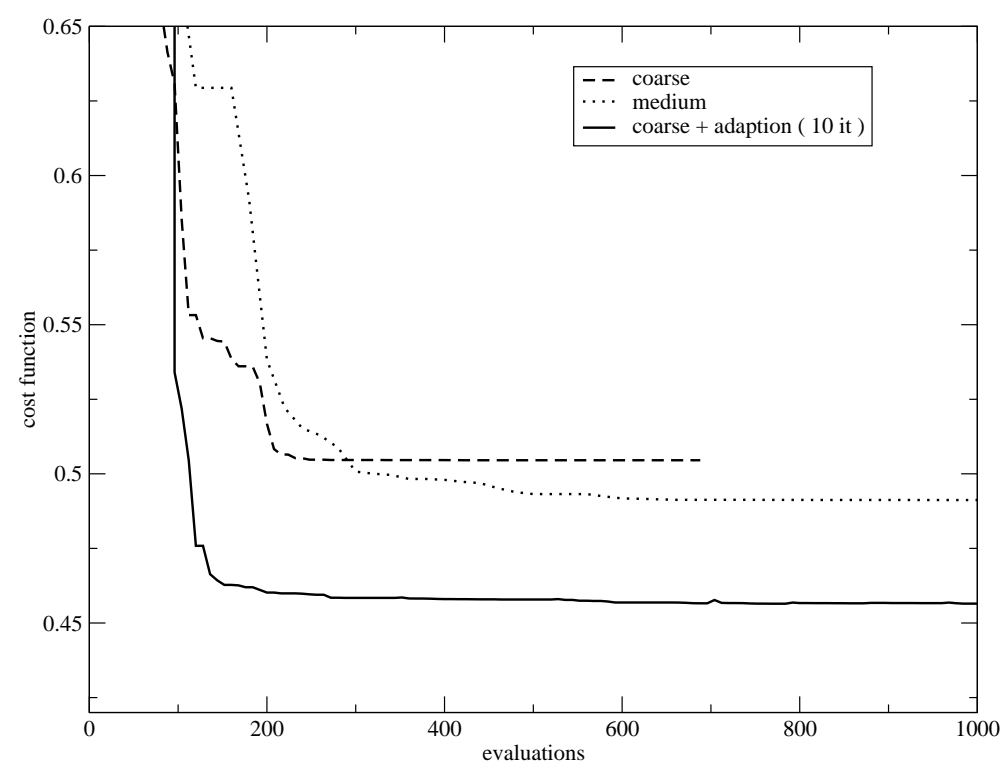

FIGURE 4. Evolution of the cost function with and without adaption using a coarse parameterization.

\section{Conclusion}

A self-adaptive parameterization methodology for shape optimization has been developed. It relies on the regularization of the design variables subject to the constraint that the shape remains unaltered in a leastsquares sense. Its efficiency has been demonstrated for a geometrical model problem using Bézier curves, and then for the aerodynamic shape optimization of a wing using the FFD approach.

The strong influence of the parameterization on the results has clearly be established. The use of the proposed adaption procedure has increased the convergence rate and improved the fitness of the shape found. 


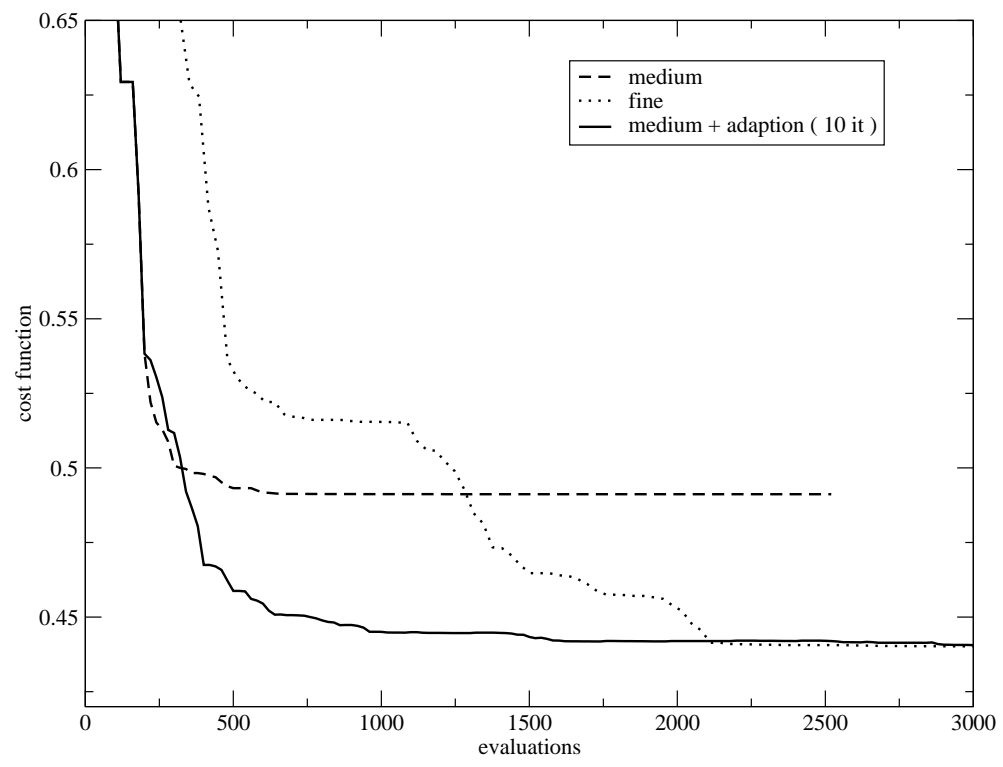

FiguRE 5. Evolution of the cost function with and without adaption using a medium parameterization.

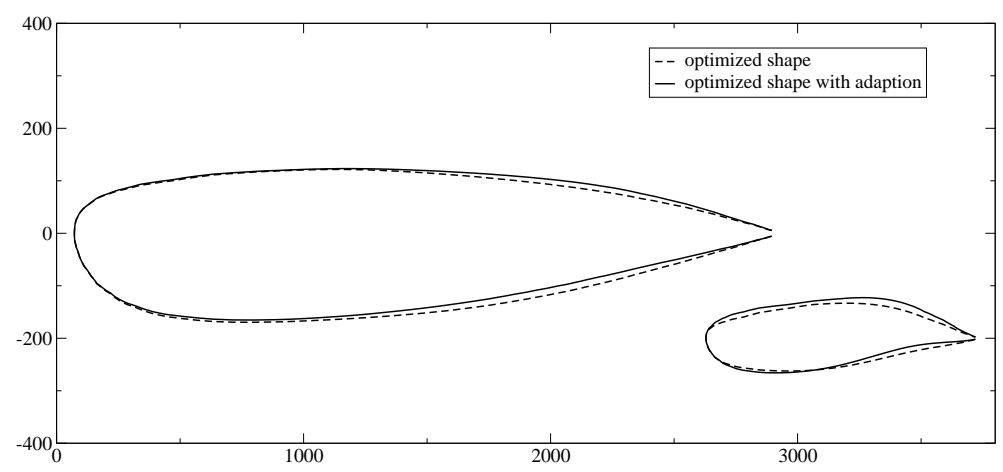

Figure 6. Comparison of the shapes obtained (root and tip section).

\section{REFERENCES}

[1] M. Andreoli, A. Janka, and J.-A. Désidéri. Free-form deformation parameterization for multilevel 3D shape optimization in aerodynamics. INRIA Research Report 5019, November 2003.

[2] J.-A. Désidéri. Two-level ideal algorithm for parametric shape optimization. Journal of Numerical Mathematics, 14, 2006.

[3] J.-A. Désidéri, B. Abou El Majd, and A. Janka. Nested and self-adaptive bézier parameterization for shape optimization. In International Conference on Control, Partial Differential Equations and Scientific Computing, Beijing, China, September 13-16 2004.

[4] J.-A. Désidéri and J.-P. Zolésio. Inverse shape optimization problems and application to airfoils. Control and Cybernatics, 34(1), 2005.

[5] R. Duvigneau. Adaptive parameterization using free-form deformation for aerodynamic shape optimization. INRIA Research Report RR-5949, July 2006.

[6] T.W. Sederberg and S.R. Parry. Free-from deformation of solid geometric models. Computer Graphics, 20(4):151-160, 1986.

[7] V. Torczon. Multi-Directional Search: A Direct Search Algorithm for Parallel Machines. PhD thesis, Houston, TX, USA, 1989. 